composition in non-alcoholic fatty liver disease (NAFLD) patients.

Methods This is a randomized, double-blind, controlled clinical trial. Twenty-nine patients were randomly assigned into probiotics and placebo groups. Multi-strain probiotics containing six different Lactobacillus and Bifidobacterium species at the concentration of 30 billion CFU were administered during the 6-month study. Anthropometric measurements like body mass index (BMI) and waist circumference (WC) were recorded. The duodenal biopsies from pre-and post-intervention were analyzed for microbiota compositions using amplicon sequencing based on the V3 region of $16 \mathrm{~s}$ rRNA.

Results The average mean age of recruited patients was $52 \pm$ 13 years. Both groups displayed the same BMI and WC at the baseline and after the intervention. The 16s rRNA analysis revealed three main prokaryotic phyla, namely Actinobacteria, Proteobacteria, and Firmicutes, with genera Streptococcus, Methylobacterium, Cutibacterium, and Prevotella being particularly common after intervention for both groups. Beta-diversity analysis of the probiotics group disclosed a significant change of gut microbiota composition upon intervention procedures $(p<0.05)$. Notably, the probiotics group also presented a decrease of Firmicutes/Bacteroidetes $(\mathrm{F} / \mathrm{B})$ ratio upon 6-month of intervention. Conversely, the placebo group illustrated a stable diversity upon intervention procedures as Alpha and Beta-diversity analyses showed no significant difference.

Conclusions We anticipated a potential pattern of dysbiosis among NAFLD patients through the high prevalence of Streptococcus in core microbiome analysis for both groups. Interestingly, we managed to obtain a decrease in Firmicutes/ Bacteroidetes ratio in the probiotics group which suggested a balance of microbial compositions through the presence of Lactobacillus and Bifidobacterium species. Hence, probiotics could be adopted as a potent preventive strategy in NAFLD patients

\section{IDDF2021-ABS-0189 METABOLIC INHIBITOR OF PFKFB3 AND ITS IMPLICATION IN IMMUNE EVASION BY UPREGULATING PD-L1 EXPRESSION VIA THE PHOSPHOPFKFB3-HIF1A-PD-L1 AXIS}

Jia-Bo Zheng*. Sun Yat-Sen University Cancer Center, China

\subsection{6/gutjnl-2021-IDDF.53}

Background PFKFB3 is a crucial metabolic enzyme which is highly upregulated in cancer cells, prompting much research on potential selective inhibitors of PFKFB3 which all have shown promising tumor inhibitive effects. However, clinical trials such as NCT02044861 on PFKFB3 inhibitors seem resultless. With recent implications of cancer metabolism and tumor immune evasion, we hypothesized that PFKFB3 inhibition and subsequent metabolic reprogramming might affect tumor immune evasion.

Methods We examined the function of PFK-15 on tumor cells in vitro and in vivo in immune-deficient nude-mice models and immune-competent mouse models such as C57/ BALB/c-CDX models and huPBMC-CDX and PBX models. CO-IP, fluorescent immunohistochemistry, CHIP and dualluciferase assays were used to investigate the underlying mechanism.
Results Inhibition of PFKFB3 using PFK-15 suppressed cell growth in human esophageal cell lines in vitro and in vivo in immune-deficient xenografts. However, inhibition of PFKFB3 caused a marked upregulation in PD-L1 which inactivated cocultured T-cells in vitro and compromised antitumor immunity in immune-competent mouse, an effect which could be reversed when PFK-15 was combined with PD-1 mab. Mechanistically, we identified ERK pathway upregulation after treatment with PFK-15, which increased PFKFB3 phosphorylation levels, causing its transformation and increased its binding with HIF-1 $\alpha$, then PhosphoPFKFB3 co-translocated into the nucleus together with HIF$1 \alpha$ by binding with importin $\alpha 5$, whereby nuclear HIF- $1 \alpha$ attaches itself to HRE regions on PD-L1 promoter, upregulating PD-L1 expression. Clinically, we observed higher Phospho-PFKFB3 in tumor tissues from non-responders to PD-1 mab treated ESCC patients.

Conclusions This study shows that inhibition of PFKFB3 increases immune evasion via the phospho-PFKFB3-HIF- $1 \alpha-$ PD-L1 axis. The translational significance of this study lies in the fact that in-vivo experiments in immune-competent mice using the closest to human immune tumor microenvironment model huPBMC-CDX/PDX combining PFKFB3 inhibitors and PD-1 mab resulted in a marked decrease in tumor development. These findings may be of relevance for the design of anti-cancer treatment trials with PFKFB3 inhibitors.

\section{IDDF2021-ABS-0196 THE EFFECT AND IMMUNE CELL ANALYSIS OF CLOSTRIDIUM BUTYRICUM ON DEXTRAN SULPHATE SODIUM INDUCED COLITIS IN MICE PRETREATED WITH ANTIBIOTIC COCKTAIL}

Jing $\mathrm{Xu}^{*}$, Haoming $\mathrm{Xu}$, Yao Peng, Hailan Zhao, Yong Zhang, Xue Guo, Jiaqi Wang Wenqi Huang, Hongli Huang, Youlian Zhou, Yuqiang Nie. Department of Gastroenterology and Hepatology, Guangzhou Digestive Disease Center, Guangzhou First People's Hospital, School of Medicine, South China University of Technology, China

\subsection{6/gutjnl-2021-IDDF.54}

Background Inflammatory bowel disease is a chronic inflammatory disease characterized by recurrent abdominal pain and diarrhea, and gut microbiota is one of its causes. Probiotics, as one of the intervention methods of microbiota, play an important role in disease treatment. This study aims to observe the effect and immune mechanism of Clostridium butyricum MIYAIRI 588 (CBM) and it's supernatant on colitis mice which pretreated with antibiotic cocktail $(\mathrm{ABx})$.

Methods Twenty four Balb/c mice aged 6-8 weeks were randomly divided into four groups, and CBM and its supernatant was used to treat colitis mice which pretreated with $\mathrm{ABx}$. The body weight, disease activity index (DAI) and colon length of mice were observed respectively. The relative expression of inflammatory cytokines in colon tissue was detected by qPCR. And the number of neutrophils, macrophages, Th1 and Th17 cells in lamina propria of mucosa was detected by flow cytometry to observe the changes of pro-inflammatory immune cells in mouse mucosa.

Results The DAI of mice were decreased, the shortened colon length was improved after CBM and its supernatant intervention (IDDF2021-ABS-0196 Figure1A. Clostridium butyricum and its supernatant ameliorated colitis and regulated immune cells; DAI scores of mice after C. Butyricum gavaging with 